\title{
Revista Colombiana de

\section{Manejo ejemplar del infarto agudo de miocardio con la utilización de protocolos sofisticados de Telemedicina}

\author{
Sameer Mehta*, Rodolfo Vega, Fredy Bojanini, Juan Corral, Álvaro Bulla, \\ Roberto Botelho, Francisco Fernández, Daniel Rodríguez, Mario A. Torres \\ y Lucas Lauar Cortizo Vidal
}

\author{
Lumen Foundation, Miami, Florida
}

Recibido el 11 de diciembre de 2017; aceptado el 12 de mayo de 2018

Disponible en Internet el 3 de octubre de 2018

\section{PALABRAS CLAVE \\ Telemedicina; Infarto agudo de miocardio; \\ Sistemas de cuidado}

\begin{abstract}
Resumen
Introducción: la Telemedicina ofrece una herramienta poderosa, rentable y escalable que permite reducir las diferencias en el tratamiento del infarto agudo de miocardio entre países desarrollados y en vía de desarrollo.

Métodos: se incorpora una estrategia en la que centros de salud en áreas remotas se conectaron estratégicamente a clínicas con salas de cateterismo 24/7. Expertos en Bogotá proporcionaron diagnóstico electrocardiográfico y tele-consulta para LATIN, activando el envío de ambulancias y la implementación de protocolos LATIN.

Resultados: un total de 113 centros en Colombia se conectaron en red cubriendo un gran segmento de la población. De los 110.823 pacientes examinados, 1.014 (1\%) fueron diagnosticados con infarto agudo de miocardio - ST. Un total de 472 (46\%) pacientes se reperfundieron, la mayoría mediante intervención coronaria percutánea, 324 (68,6\%). Las principales razones para el no tratamiento incluyeron rechazo de seguro médico, falta de unidades de cuidados intensivos y dolor torácico > 12 horas. El tiempo de diagnóstico fue de 5.5 con una precisión del $98 \%$.

Conclusiones: LATIN demuestra la posibilidad de crear una estrategia para tratar el infarto agudo de miocardio con base en la población y guiada por Telemedicina, la cual expande el acceso al tratamiento de emergencia en países en vía de desarrollo, en los cuales ésta adquiere importantes implicaciones en salud pública como una aproximación global al cuidado urgente de esta afección.

(C) 2018 Sociedad Colombiana de Cardiología y Cirugía Cardiovascular. Publicado por Elsevier España, S.L.U. Este es un artículo Open Access bajo la licencia CC BY-NC-ND (http:// creativecommons.org/licenses/by-nc-nd/4.0/).
\end{abstract}

\footnotetext{
* Autor para correspondencia.

Correo electrónico: dannrov@gmail.com (S. Mehta).
} 


\section{KEYWORDS}

Telemedicine;

Acute myocardial

infarction;

Health care systems
An acute myocardial infarction management model using advanced Telemedicine protocols

\begin{abstract}
Introduction: Telemedicine offers a powerful and cost-effective tool that uses scales in order to reduce the differences in the treatment of acute myocardial infarction between the developed and developing countries.

Methods: A strategy was used, in which health centres in remote areas were strategically connected to clinics with 24/7 catheter laboratories. Experts in Bogota provided the electrocardiographic diagnosis and tele-consultation using the Latin America Telemedicine Infarct Network (LATIN), activating the sending of ambulances, and the implementation of the LATIN protocols.

Results: A total of 113 centres in Colombia were connected to a network covering a large segment of the population. Of the 110,823 patients examined, $1014(1 \%)$ were diagnosed with an acute ST-myocardial infarction. A total of $472(46 \%)$ of these patients were reperfused, with the majority $(324,68.6 \%)$ by means of percutaneous coronary intervention. The main reasons for non-treatment included refusal of medical insurance, lack of intensive care units, and chest pain > 12 hours. The time to diagnosis was 5.5 minutes with an accuracy of $98 \%$.

Conclusions: LATIN demonstrates the possibility of creating a strategy for treating acute myocardial infarction in a population base and guided by Telemedicine. This widens the access to emergency treatment in developing countries, where this acquires significant implications in public health, such as an overall approach to the urgent treatment of this condition.

(c) 2018 Sociedad Colombiana de Cardiología y Cirugía Cardiovascular. Published by Elsevier España, S.L.U. This is an open access article under the CC BY-NC-ND license (http:// creativecommons.org/licenses/by-nc-nd/4.0/).
\end{abstract}

\section{Introducción}

Las guías de manejo actualizadas del infarto agudo de miocardio con elevación del ST del Colegio Americano de Cardiología y la Sociedad Europea de Cardiología enfatizan la necesidad de desarrollar sistemas de tratamiento para el acceso a la intervención coronaria percutánea primaria $(\mathrm{PCl}$, su sigla en inglés) $)^{1-6}$. Disminuir los tiempos puerta-balón (DBT) se ha convertido en el "mantra" de la PCl primaria ya que existe una correlación directa entre la reducción del DBT y la mortalidad a corto y largo plazo ${ }^{7-9}$. El empleo de estas estrategias documentadas en las guías para reducir el DBT ha demostrado resultados a largo plazo y a su vez rentabilidad ${ }^{10-13}$

El aumento en la utilización de la Telemedicina ha recibido recientemente recomendaciones por parte del Colegio Americano de Médicos ${ }^{18,19}$, basadas en recomendaciones de investigaciones relevantes que incluyeron un metaanálisis de 5.407 artículos, con 8.904 pacientes de Europa, Asia, América del Norte y América del Sur. En este metaanálisis, el uso de telemedicina se asoció con una reducción estadísticamente significativa en la mortalidad hospitalaria, a los 30 días y al año ${ }^{19}$. Igualmente, diferentes investigadores han señalado una reducción en el tiempo de isquemia para los pacientes transferidos con infarto agudo de miocardio - ST mediante el uso de redes de teléfonos inteligentes ${ }^{20}$, sistemas de computación y datos en nube ${ }^{21}$; de igual manera, el triage prehospitalario se redujo a la mitad con el uso de la telemedicina ${ }^{22}$. El aumento en el acceso a estos servicios es particularmente beneficioso para los pacientes rurales y para reducir las diferencias de atención entre los países desarrollados y aquellos en vía de desarrollo ${ }^{23-26}$.

Sumado a lo anterior, últimamente en muchos otros países como Polonia, Italia, Brasil y Canadá27-33, se ha evidenciado un aumento en las redes de infarto agudo de miocardio - ST (infarto agudo de miocardio con elevación del ST) guiadas por Telemedicina.

La telemedicina ha demostrado aumentar dramáticamente el acceso a la atención en salud, como lo demostraron Rasmussen et al. ${ }^{34}$, quienes determinaron que el $81 \%$ de su población podría tratarse en menos de 120 minutos, incluso con una distancia mayor a $95 \mathrm{Km}$. En Dinamarca, Sørensen et al. ${ }^{35}$ tomaron en cuenta si el paciente pertenecía a una comunidad rural o urbana. Se incluyó una muestra de 759 pacientes, donde el porcentaje de aquellos con un diagnóstico prehospitalario que lograron un DTB menor a 120 minutos fue del $86 \%$, frente a un $32 \%$ de aquellos que no tenían diagnóstico $(p<0,001)$. Como resultado de estas iniciativas, la mortalidad total en un rango de 4.3 años de seguimiento fue del $18 \%$ en pacientes con diagnóstico prehospitalario frente al $31 \%$ en pacientes sin este $(p=0,003)^{35}$.

El uso de telemedicina para el diagnóstico electrocardiográfico pre-hospitalario puede reducir tanto el tiempo puerta - aguja (DTN) como el DTB. Utilizando protocolos efectivos de telemedicina, el trabajo de Sánchez-Ross $M$, et $\mathrm{al}^{36}$ mostró una reducción en DTB $(63 \mathrm{~min}$ frente a $119 \mathrm{~min}$ en el control, $p<0,00004)$, menor pico de troponina $(39,5 \mathrm{ng}$ / $\mathrm{ml}$ frente a 87,6 $\mathrm{ng} / \mathrm{ml}, \mathrm{p}=0,005)$, fracciones de eyección ventricular más altas (50\% frente a $35 \%, p=0,004)$ y menor tiempo de hospitalización ( 3 días frente a 5,5 días, $\mathrm{p}$ 
$<0,001)$. En un trabajo similar, Dallan et al. ${ }^{26}$ determinaron que el DTB utilizando el protocolo LATIN fue de 32 minutos en comparación con 85 minutos mediante el protocolo anterior $(p<0,05)$.

Las investigaciones más recientes que emplearon protocolos de telemedicina, han cuantificado el impacto económico de estas estrategias. Yoculan et al. ${ }^{30}$, determinaron que en un posible escenario donde la tasa de $\mathrm{PCl}$ aumenta de 19 al $60 \%$, el ahorro podría ser de $\$ 13$ millones USD, debido a la reducción del costo indirecto en mortalidad, morbilidad y tratamiento farmacológico. Igualmente, Brunetti et al. ${ }^{33}$, demostraron que con el uso de la telemedicina, el ahorro por consulta de electrocardiograma puede ser de $€ 8.10 \mathrm{a} € 38.41$, y se podrían salvar 69 vidas por año con un costo de $€ 1.927$ año de vida-ajustado.

En nuestros anteriores trabajos, se ha reportado extensamente la utilización de la telemedicina para reducir la diferencia de atención entre los países desarrollados y en vía de desarrollo. Ahora se informa sobre el impacto de un programa de infarto agudo de miocardio basado en la población y guiado por telemedicina: Red de infarto de telemedicina de América Latina (LATIN por su sigla en inglés). El programa es una iniciativa de salud pública en expansión, que incorpora protocolos sofisticados de telemedicina y se ha utilizado para clasificar más de 400.000 pacientes en Colombia, Brasil y México. En esta publicación, se exponen la metodología y los resultados de este programa en Colombia.

\section{MÉTODOS}

\section{Visión general del estudio}

En algunas regiones de Colombia se implementó un protocolo estandarizado "LATIN", en las cuales se estableció un modelo de Hub y Spokes, con la meta de expandir el acceso a la $\mathrm{PCl}$ mejorando la efectividad y los resultados en los pacientes con infarto agudo de miocardio - ST. En cada región se identificaron centros con disponibilidad 24/7 para la realización de angioplastia primaria ('Hubs'), donde se llevaron a cabo procedimientos de $\mathrm{PCl}$ en pacientes con infarto agudo de miocardio - ST referidos desde los centros de salud y hospitales de primer o segundo nivel de atención ('Spokes') de la región. De igual manera, dentro del programa se establecieron tres centros principales para proveer diagnóstico electrocardiográfico y Teleconsulta de alta calidad para toda la red. Se implementó una plataforma de telemedicina en cada centro con la cual se creó una base de datos con toda la información. Se recolectaron datos sobre las estrategias de manejo de pacientes, resultados y costos, y se emprendió el análisis estadístico de los mismos, comparándolos con información histórica de costos y de mortalidad, determinando el éxito del programa.

\section{Selección de Colombia}

La tabla 1 resalta algunas de las características que fueron esenciales al seleccionar a Colombia como el sitio inicial para demostrar la viabilidad de la telemedicina en disminuir las diferencias existentes en el manejo del infarto agudo de miocardio. Esta tabla compara Estados Unidos, Brasil,
México y Colombia en áreas relevantes al cuidado del infarto agudo de miocardio. Colombia es un país menos urbanizado y con una mayor proporción de su población viviendo bajo niveles de pobreza. La mortalidad por enfermedades cardíacas es alta y el presupuesto del sector salud es bajo; además, el acceso al personal médico y camas hospitalarias es considerablemente más bajo que en los Estados Unidos.

Con la mayoría de las instalaciones médicas bien equipadas, incluyendo los centros para $\mathrm{PCl}$ ubicados en áreas urbanas, el acceso al cuidado de la salud de calidad y ambulancias confiables es problemático para los residentes rurales.

\section{Estrategia de Hub y Spokes}

Para expandir el acceso se formuló una estrategia de Hub y Spokes. Con esta estructura, centros de salud y hospitales de primer o segundo nivel de atención en áreas remotas ("Spokes") fueron conectados de manera estratégica a centros especializados ("Hubs") que contaran con instalaciones para la realización de $\mathrm{PCl}$. La figura 1 demuestra la configuración de Hub y Spokes de LATIN en Colombia. Las metas fueron crear una estrategia eficiente para Triage, Telemedicina y Transporte ( $3 \mathrm{~T}$ ) en cada Spoke y contar con la disponibilidad de instalaciones para $\mathrm{PCl}$ 24/7 en cada Hub. La creación de este programa masivo en Colombia fue particularmente desafiante, debido a que algunas regiones del país fueron logísticamente difíciles de incluir en la red (fig. 2). A lo anterior se suma la localización de numerosos centros en terrenos geográficamente remotos; pese a estas dificultades, la cobertura de LATIN en Colombia para manejo del infarto agudo de miocardio ha aumentado, logrando que gran parte de los 48 millones de habitantes, tengan acceso a las distintas modalidades de atención del infarto agudo de miocardio guiadas por telemedicina. La falta de servicios de ambulancia y la escasez de camas en la UCI fueron el mayor desafío logístico. Se instauraron mecanismos de retroalimentación de manera exitosa dentro de todos los sitios LATIN.

\section{Infraestructura de telemedicina}

Cardiólogos expertos ubicados en una central en Bogotá, suministraron diagnóstico electrocardiográfico urgente y Teleconsulta 24/7 durante todo el proceso; al realizar el diagnóstico de infarto agudo de miocardio - ST activaron el envío de ambulancias e iniciaron los protocolos LATIN y sus sistemas y redes de tratamiento. En estos sitios se instaló una plataforma sofisticada de telemedicina, la cual suministra diagnóstico inmediato, aseguramiento de red, interfaz de usuario y compatibilidad, aseguramiento de calidad y manejo de la base de datos. Al emplear una combinación de redes de Internet, fax, modem y teléfono, esta plataforma permitió la vectorización automática del electrocardiograma, la eliminación de artefactos y la incorporación del electrocardiograma en el registro médico. El software de comunicación generó alertas automáticas desde la ubicación del cardiólogo remoto tanto a Hubs como a Spokes, y fue rutinariamente utilizado para dar retroalimentación. El entrenamiento y reentrenamiento riguroso de los cardiólogos remotos en los procesos y procedimientos de LATIN es un ejercicio continuo. 
Tabla 1 Diferencias económicas y de salubridad entre países desarrollados y en vía de desarrollo

\begin{tabular}{|c|c|c|c|c|c|}
\hline Indicador & & USA & Brasil & Colombia & México \\
\hline Población (millones) & & 323.1 & 207.7 & 48.6 & 127.5 \\
\hline Población urbana (\%) & & 82.5 & 86.0 & 77.4 & 79.8 \\
\hline Ingreso per cápita (USD) & & 57.220 & 15.646 & 14.164 & 17.880 \\
\hline Gasto nacional anual en salud como proporción de GDP (\%) & & 17.1 & 8.3 & 7.2 & 6.3 \\
\hline Gasto total en salud per cápita (US\$) & & 9.403 & 1.318 & 962 & 1.122 \\
\hline Relación de número de personas en pobreza a $\$ 3,1$ por día PPP (\%) & & - & 7.6 & 13.2 & 10.9 \\
\hline Relación de médicos (por 100.000 hab.) & & 255 & 185 & 156 & 207 \\
\hline Relación de camas hospitalarias (por 100.000 hab.) & & 326 & 230 & 160 & 168 \\
\hline Total de labs. de cateterización & & 2.000 & 799 & 245 & 266 \\
\hline Total PCl/año & & 400.000 & 150.000 & 45.000 & 95.000 \\
\hline Número total de ambulancias (por 100.000 hab.) & & 48,3 & 10,9 & 4,8 & 6,4 \\
\hline Tasa de mortalidad por enf. cardiaca isquémica (por 100,000 hab.) & & 114,8 & 114,1 & 80,5 & 90,0 \\
\hline \multirow[t]{2}{*}{ Tasa de mortalidad estimada por enf. cardiaca isquémica } & 45 - 64 años & 85,38 & 102,40 & 103,01 & 101.06 \\
\hline & $>65$ años & 749,55 & 518,77 & $1.008,30$ & 968.30 \\
\hline
\end{tabular}

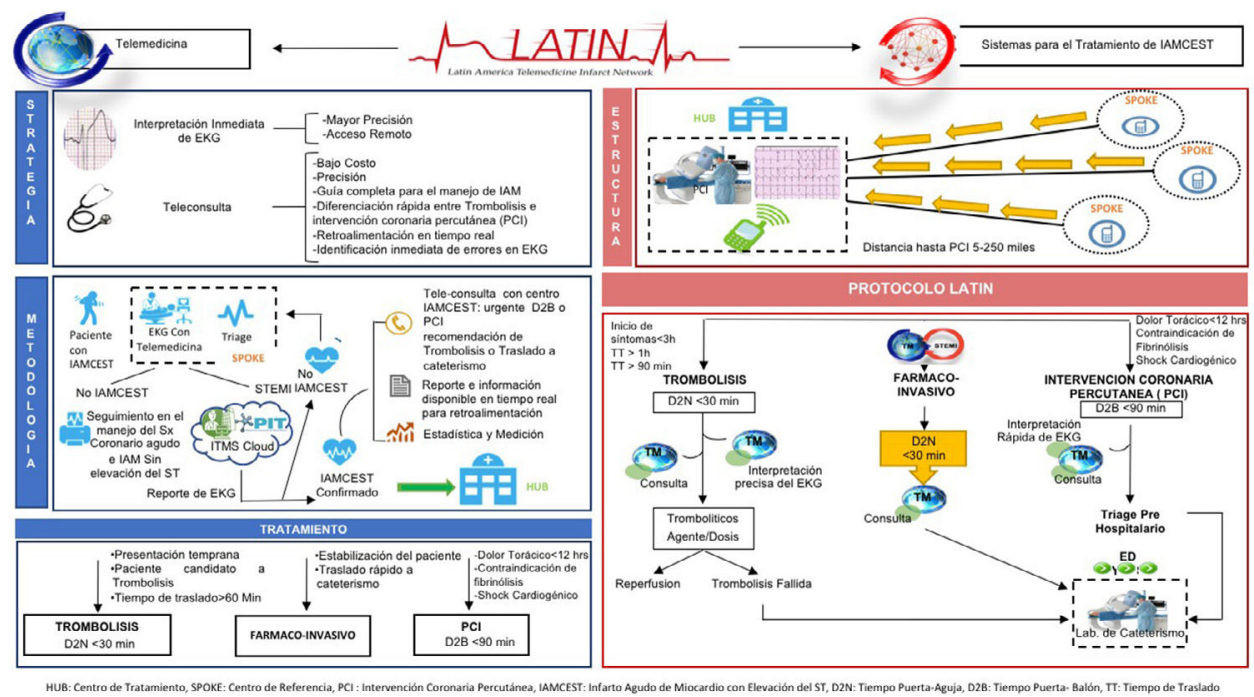

Figura 1 Protocolo LATIN.

\section{Protocolo LATIN estandarizado}

Con base en la última actualización de las guías para el manejo del infarto agudo de miocardio - ST, se creó un protocolo LATIN estandarizado (fig. 3) con el fin de suministrar un manejo integral del infarto agudo de miocardio. La responsabilidad primaria de los Hubs fue llevar a cabo intervenciones para el infarto agudo de miocardio - ST con tiempos puerta-balón (D2B) cortos. Los Spokes (un promedio de hasta 5 por Hub) ubicados entre 8 y 100 kilómetros de cada Hub, administraron terapia trombolítica a pacientes elegibles y agilizaron la transferencia a los Hubs. Cada Spoke de LATIN tenía directrices avanzadas para el tratamiento de pacientes con infarto agudo de miocardio por medio de trombólisis o intervención coronaria percutánea $(\mathrm{PCl})$ con base en su localización y distancia hasta el Hub. En general, según lo ilustrado en la figura 1 , se recomendó la terapia trombolítica para la presentación temprana ( 3 horas a partir del inicio del dolor), mientras que se abogó por angioplastia primaria (ATCP) cuando la transferencia a un Hub estuviera fácilmente disponible. La lisis fallida fue una indicación absoluta para transferencia al Hub para angioplastia de rescate. Todos los pacientes con trombólisis exitosa fueron transferidos al Hub dentro de un periodo razonable (4 24 horas).

\section{Entrenamiento de Hubs y Spokes}

Para los Spokes se empleó una estrategia de triage, Telemedicina y transporte $(3 \mathrm{~T})$ como mandatos críticos. Esta disciplina fue instituida rigurosamente en todos los Spokes. Se hizo énfasis en la obtención inmediata (dentro de los siguientes 5 minutos) de un electrocardiograma y su transmisión para diagnóstico por telemedicina. Los retrasos de transporte fueron endémicos en casi todos los Spokes, 


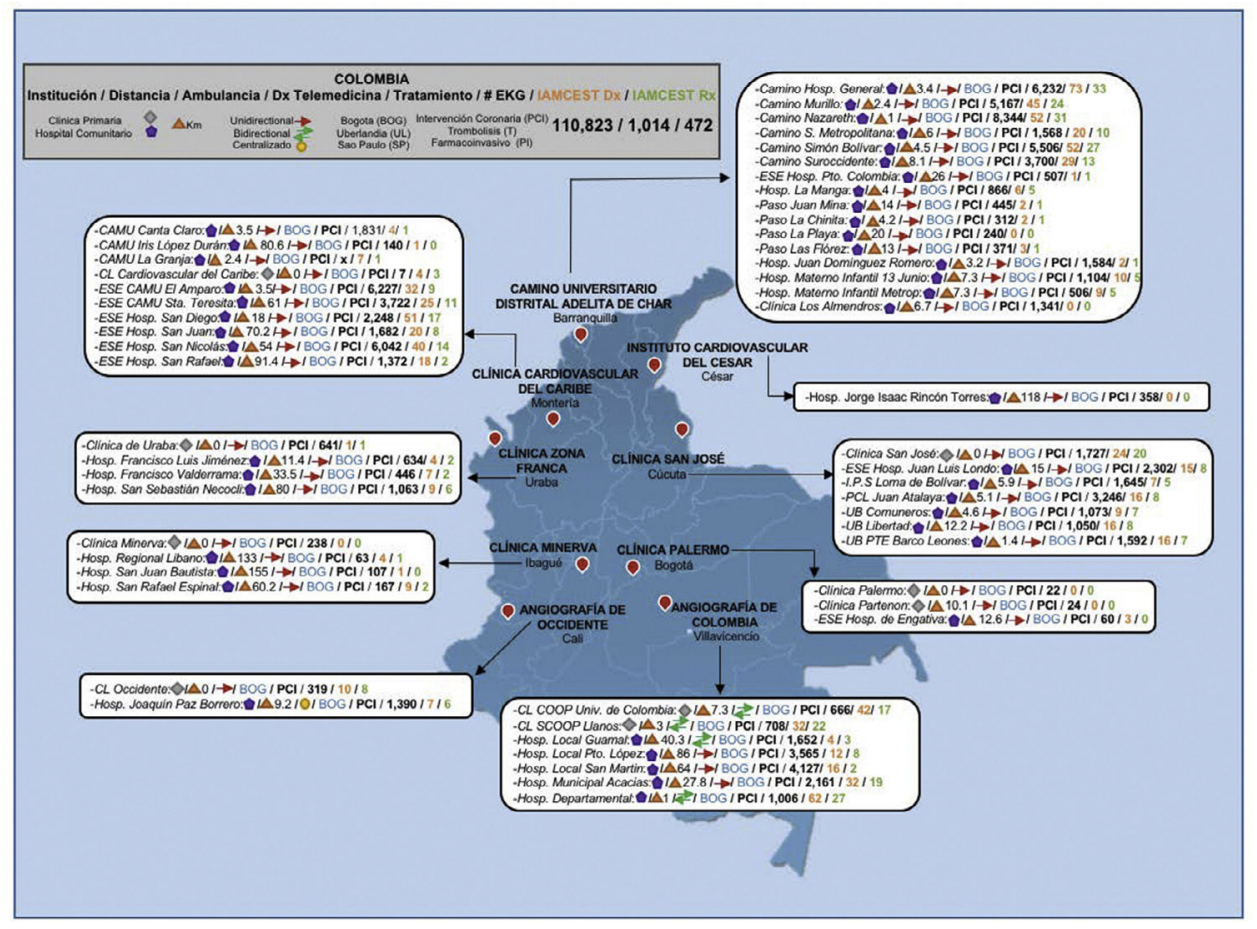

Figura 2 Mapa Logístico LATIN Colombia.

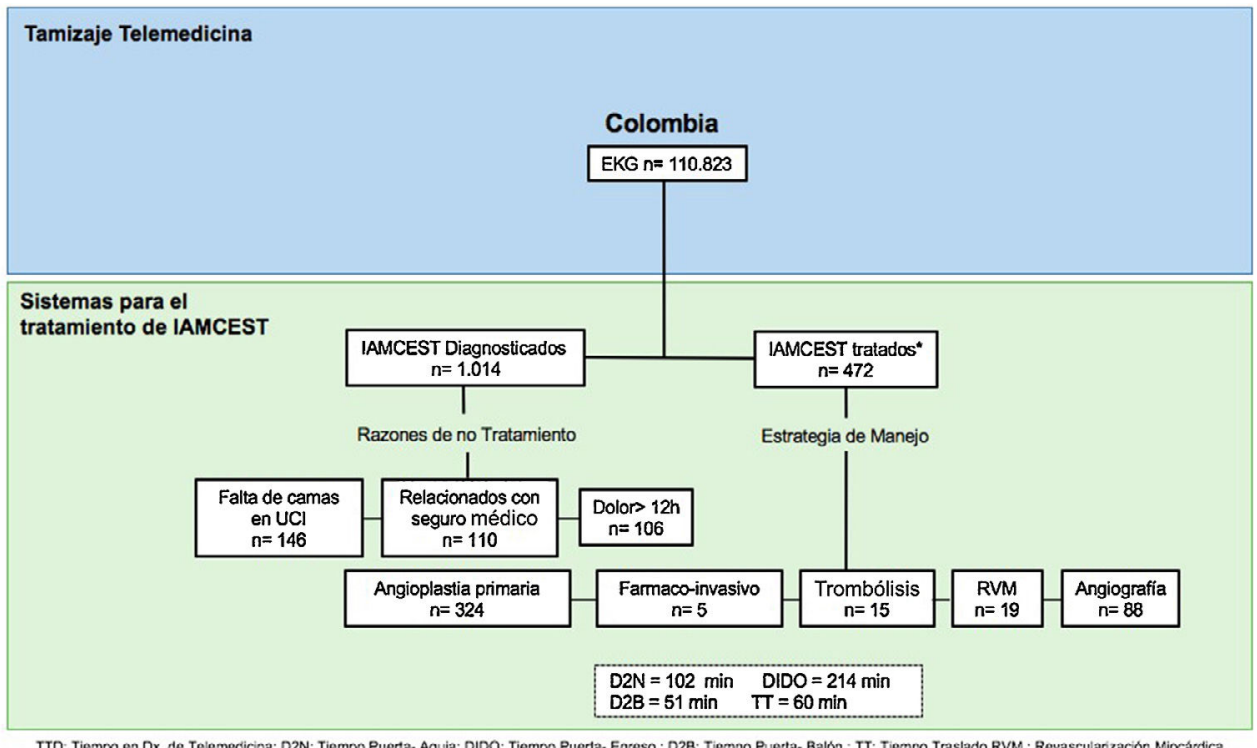

Figura 3 LATIN Colombia Diagrama de Flujo.

y fueron desafíos continuos. Para obviarlos se establecieron comunicaciones tempranas con servicios médicos de emergencia (EMS) para cada paciente con infarto agudo de miocardio, con la finalidad de facilitar su traslado en ambulancia. En los Hubs, el énfasis estuvo en el trabajo en equipo, evitar el servicio de emergencia, disponibilidad 24/7, alerta de llamada única y equipos internos, siempre que fuese posible, al igual que en la retroalimentación. El manejo de trombos, vasodilatadores intracoronarios, shock y lesiones no culpables fue un asunto de entrenamiento continuo.

\section{Base de datos y criterios de desempeño}

La base de datos LATIN proveyó la captura de importantes métricas para medir individualmente y referenciar los tres componentes esenciales de la ruta LATIN - Spokes, Hubs y Sistemas de Telemedicina. Con esta metodología, se empleó tiempo puerta ingreso - puerta egreso (DIDO "Door In - Door Out") y tiempo medio de transferencia (TT) para medir la funcionalidad del Spoke; los tiempos puerta-balón (D2B) para evaluar el desempeño del Hub y el tiempo hasta el 
Tabla 2

\begin{tabular}{ll}
\hline Parámetro & Colombia \\
\hline Hubs & 16 \\
Spokes & 97 \\
Transmisiones de EKG & 110.823 \\
Infarto de miocardio con & 1.014 \\
$\quad$ elevación de ST & \\
(diagnóstico remoto por & \\
Telemedicina) & \\
Masculinos (\%) & 65,98 \\
Femeninos (\%) & 34,02 \\
Diabetes mellitus & $174(17,16 \%)$ \\
Fumar & $202(19,92 \%)$ \\
HTN & $487(48,03 \%)$ \\
Infarto de miocardio con & $472(46,54 \%)$ \\
elevación de ST (tratado) & \\
Estrategia de tratamiento & $15(3,17 \%)$ \\
a. Trombólisis & $5(1,05 \%)$ \\
b. Fármaco-invasiva & $324(68,64 \%)$ \\
C. PCI Primaria & $19(4,02 \%)$ \\
d. CABG & $88(18,6 \%)$ \\
e. Angiografía & \\
Mortalidad total & $42(4,2 \%)$ \\
En Spoke & $21(50,0 \%)$ \\
Transferencia & $10(23,8 \%)$ \\
En Hub & $11(26,2 \%)$ \\
\hline
\end{tabular}

diagnóstico por Telemedicina (TTD "Time to Telemedicine Diagnosis") para cuantificar la eficiencia de la telemedicina.

\section{RESULTADOS}

\section{Configuración de la red}

La configuración de Hubs y Spokes de LATIN se muestra en la figura 1. Este diagrama corresponde a una ilustración estratégica de 16 Hubs y 97 Spokes, la logística de telemedicina, la estructura de ambulancias y los detalles de transferencias de Spoke a Hub. El número medio de Spokes/Hub fue de 6,1 (rango de 1 - 17). Barranquilla presentó una mayor relación de Spokes/Hub que otros sitios. Entre los Spokes, $81,8 \%$ eran hospitales comunitarios, y $18,2 \%$ eran centros de salud primarios y consultorios pequeños. Cardiólogos expertos revisaron los electrocardiogramas de 110.823 pacientes. La distancia media entre Hubs y Spokes fue de $24,1 \mathrm{Km}$ (rango de 0 a $100 \mathrm{Km}$ ). El transporte de ambulancia fue el siguiente: Unidireccional 49 (Spoke - Hub), Bidireccional 9 (Hub - Spoke - Hub), Centralizados 2.

\section{Datos demográficos y diagrama de flujo del tratamiento LATIN}

La mayoría de pacientes con infarto agudo de miocardio - ST tratados, consta de individuos masculinos con factores de riesgo esperados como diabetes y tabaquismo, en 17 y $19 \%$ respectivamente, pero con mayor índice de hipertensión (48\%). La tabla 2 ilustra los datos demográficos de estos pacientes. Los pacientes tamizados eran casi una década más jóvenes que los tratados - para la cohorte de 110.823 pacientes la media de edad fue de 52 años y para los pacientes reperfundidos fue de 62 años. La figura 3 ilustra el diagrama de flujo detallando la ruta de los pacientes LATIN que comienza con su tamización por electrocardiograma hasta el tratamiento definitivo para el infarto agudo de miocardio - ST. Entre el $1^{\circ}$ de abril de 2014, hasta el presente, 110.823 pacientes fueron tamizados con base en su electrocardiograma de presentación. De estos 110.823 electrocardiogramas que fueron evaluados de manera remota y urgente por parte de cardiólogos expertos, 1.014 (1\%) cumplieron criterios para un diagnóstico de infarto agudo de miocardio - ST y constituyeron una alerta de telemedicina simultánea tanto para el Hub como para el Spoke. Un total de 472 pacientes $(46,54 \%$ de pacientes con diagnóstico de infarto agudo de miocardio - ST) se sometieron a reperfusión urgente.

\section{Estrategia de reperfusión y funcionalidad del proceso}

De los 1.014 pacientes (1\% de los tamizados) con infarto agudo de miocardio - ST confirmado, 472 (46,54\%) fueron reperfundidos: angioplastia primaria (ACTP) 324 (68,6\%), angiografía $88(18,6 \%)$, cirugía de revascularización miocárdica (RVM) 19 (4\%), fármaco-invasivo 5 (1\%) y trombólisis 15 (3\%). Se dividieron los resultados del proceso en tres grupos: a) Funcionalidad del Spoke - determinada por medio del tiempo puerta-aguja (D2N), 58 minutos; tiempo puerta ingreso - puerta egreso (DIDO), 267 min; y tiempo de transferencia (TT) 55 minutos; b) Funcionalidad de Telemedicina Tiempo hasta diagnóstico por telemedicina (TTD), 5,8 minutos; este es el tiempo medio que existe entre la transmisión del electrocardiograma al centro de telemedicina y hasta la recepción de un diagnóstico confirmatorio en el Spoke; c) Funcionalidad del Hub - Tiempo Puerta-Balón, 95 minutos.

\section{Razones para no tratamiento (tabla 2)}

De las numerosas razones para no tratamiento, las tres más comunes fueron: dolor de pecho mayor a 12 horas, falta de camas en la $\mathrm{UCl}$ y negaciones del seguro. Este patrón fue reconocido de manera uniforme a través de la mayoría de Spokes y Hubs.

\section{Mortalidad (tabla 3)}

La mortalidad total intrahospitalaria fue de 42 pacientes (4,6\%); de las cuales se presentaron 21 en los Spokes (50\%), 10 durante el traslado $(23,8 \%)$ y 11 en el Hub $(26,2 \%)$.

\section{Discusión}

LATIN demuestra un enfoque sistemático guiado por telemedicina para la atención poblacional del infarto agudo de miocardio, el cual es eficiente y escalable al aumentar la cobertura. Aunque la telemedicina se ha utilizado previamente para el manejo del infarto agudo de miocardio, LATIN es la primera red estandarizada de atención de infarto agudo de miocardio - ST basada en población que ha utilizado 
Tabla 3

\begin{tabular}{ll}
\hline Parámetro & Colombia \\
\hline Tiempo puerta-aguja (D2N) - min & 58 \\
Tiempo puerta-balón (D2B) - min & 95 \\
Puerta ingreso - puerta egreso (DIDO) & 267 \\
$\quad$ - min & \\
Tiempo medio de transferencia (min) & 55 \\
Tiempo hasta diagnóstico por & 4,5 \\
$\quad$ telemedicina (TTD) - min & \\
\hline
\end{tabular}

metódicamente la telemedicina para cubrir grandes urbes y guiar el tratamiento de dicha afección

Los problemas fundamentales han sido evaluados. El más importante es el diagnóstico erróneo del infarto agudo de miocardio - ST en aproximadamente un tercio de los pacientes ${ }^{14}$, ocasionando un mal manejo clínico y una exposición innecesaria a investigaciones o tratamientos potencialmente peligrosos ${ }^{15}$. Además, la lectura computarizada del electrocardiograma $(\mathrm{CIE})$ no es una solución confiable $^{15}$ ya que sobreinterpreta los hallazgos. Estas limitaciones se superan con la digitalización ${ }^{16}$ y la telemedicina, haciendo un uso remoto e inteligente de la combinación de robótica y telecomunicaciones ${ }^{17,18}$. Esto permite la confirmación electrocardiográfica por expertos como se recomienda repetidamente en las publicaciones ${ }^{15}$. Al diseñar LATIN, nos enfocamos inicialmente en optimizar la precisión del diagnóstico electrocardiográfico mediante cardiólogos expertos. Durante el período de estudio, la interpretación electrocardiográfica remota aumentó su eficiencia, reduciendo el tiempo de diagnóstico en telemedicina (TTD) de 12 minutos a menos de 6 minutos.

LATIN demuestra una utilización pragmática de plataformas de telemedicina para reducir las barreras de acceso a la atención de infarto agudo de miocardio existentes en los países en vía de desarrollo. La combinación innovadora de telemedicina y sistemas de atención del infarto agudo de miocardio - ST permite que la mayoría de pacientes con infarto agudo de miocardio sean tratados mediante $\mathrm{PCI}$. La pequeña cohorte tratada con trombólisis puede atribuirse principalmente a la presentación tardía de los pacientes (donde los trombolíticos serían ineficaces) y a la dependencia de los agentes de primera generación (menos efectivos y con más complicaciones). Además, desde el inicio, LATIN se enfocó minuciosamente en la transferencia de pacientes en ambulancias, asegurando que la $\mathrm{PCl}$ pudiera realizarse a tiempo en la mayoría de los sitios. El DTN aumentado, probablemente sea el resultado de una estrategia impopular y de su poca utilización.

Desde el inicio de LATIN, se identificó que el rendimiento de los Spokes era clave para su éxito. Gradualmente se capacitó cada Spoke, incorporando una estrategia de "3T"': triage, telemedicina y transporte para mejorar los parámetros de puerta-entrada puerta-egreso (DIDO) y tiempo de transferencia (TT). El pequeño tamaño de los Hubs permitió mayor agilidad en el paso por las salas de urgencias, contribuyendo a reducir los tiempos de D2B. La activación de un solo sistema, los estrictos horarios de informes con tiempos obligatorios, la comunicación efectiva, el trabajo en equipo y la retroalimentación también contribuyeron a la eficiencia de los centros.

Sin embargo, LATIN reveló deficiencias importantes del sistema que fueron resistentes a la mejoría, disminuyendo el impacto general del programa en el manejo del infarto agudo de miocardio. Un porcentaje considerable de pacientes diagnosticados con infarto agudo de miocardio - ST no fueron tratados; las principales razones fueron la presentación tardía de los pacientes a los centros, la falta de camas en las $\mathrm{UCl}$ y negación en el servicio por parte de las aseguradoras. Al explorar soluciones, consideramos que una mayor difusión de los resultados de LATIN y el uso apropiado de las redes sociales mejorarán estos tres problemas. Aún así, el efecto de una gran proporción de pacientes no tratados disminuye la rentabilidad de LATIN y el poder de detección de la telemedicina.

Otra limitación de esta investigación ha sido que en la selección de pacientes, no se utilizaron pre-probabilidades en términos de factores de riesgo coronario. Con la expansión de LATIN a otras regiones de Brasil, Colombia, México, Chile y Perú el proceso de selección de LATIN integrará criterios más estrictos.

Los autores consideran que la corrección de las deficiencias anteriores puede ofrecer una estrategia altamente efectiva para proporcionar un tratamiento estandarizado de calidad en el infarto agudo de miocardio aplicable a grandes poblaciones. Además, los expertos en centros remotos que utilizan plataformas de telemedicina pueden guiar de manera rentable el manejo del infarto agudo de miocardio. También se anticipa una mejora en la tecnología de telemedicina a través del aprendizaje en el manejo de los equipos, la realidad aumentada y la tecnología en la nube. LATIN, con estas mejoras, puede servir como una plantilla para tratar el infarto agudo de miocardio en países de ingresos medianos y bajos.

En lo que respecta a Colombia, se cree firmemente que LATIN ha mejorado el manejo del infarto agudo de miocardio por cuanto:

1. Se ha concientizado a los pacientes en el infarto agudo de miocardio. LATIN es un programa monumental el cual ha interactuado con numerosas partes en distintos niveles: pacientes, médicos generales, cardiólogos, administradores de hospitales, médicos de urgencias, paramédicos, prensa y medios de comunicación. Para todos estos constituyentes y beneficiarios de LATIN, existe un mensaje inequívoco de educación sobre numerosos aspectos del manejo del infarto agudo de miocardio. Esto incluye el reconocimiento temprano de los síntomas y la urgencia de buscar atención hospitalaria. Se han resaltado los avances de la $\mathrm{PCl}$ y se ha enfatizado la importancia en la transferencia realizada por ambulancias.

2. LATIN Colombia ha expandido enormemente el acceso a la atención de infarto agudo de miocardio de calidad en regiones remotas y pobres del país. Esto ha ocurrido a través de la unión de la telemedicina con un sistema de Hub y Spoke. Esta estrategia en particular se inspiró en Level 1 Heart Attack Program de Minnesota, iniciado por el Doctor Timothy Henry; RACE, en Carolina del Norte, por los doctores Christopher Granger y Jamie Jollis; y PRINCE, por el Doctor Sameer Mehta en Puerto Rico. 
Sofisticados programas de telemedicina complementados con tecnología en la nube, navegación por GPS y aprendizaje de la tecnología han elevado la atención del infarto agudo de miocardio en Colombia. El éxito de las redes con tecnología avanzada ha impulsado a Colombia a convertirse en un líder en el manejo del infarto agudo de miocardio en Suramérica.

3. Los pacientes más vulnerables, de bajos recursos y ubicados en áreas rurales, sin acceso previo a la reperfusión temprana para el infarto agudo de miocardio han tenido acceso al tratamiento indicado por las guías.

4. Las diferentes alternativas de manejo para los pacientes con infarto agudo de miocardio en Colombia han experimentado un cambio notable hacia la $\mathrm{PCl}$ con la instalación de LATIN. Se observó un aumento estadísticamente significativo en la utilización de $\mathrm{PCl}$ y una reducción correspondiente en la mortalidad.

5. LATIN ha contribuido a mejoras cualitativas y cuantitativas en el servicio de ambulancias en Colombia. Se han establecido protocolos de alerta prehospitalarios además de aquellos para evadir el servicio de urgencias, aunque todavía se requiere mucho trabajo. La capacitación de paramédicos está mejorando y cada vez más pacientes tienen acceso a la atención de ambulancias para llegar oportunamente a los hospitales.

6. El "efecto halo" en la gestión científica de calidad, basada en las guías del infarto agudo de miocardio ha comenzado a mejorar los procesos y el procedimiento para el tratamiento del infarto agudo de miocardio ST en Colombia. LATIN es un ejemplo de combinación de trabajo en equipo eficiente, estructura y dinámica, necesarios para gestionar con éxito un programa de tal magnitud. Su influencia ya se evidencia en numerosos hospitales cercanos a los sitios LATIN.

7. LATIN ha estimulado la práctica de mejores procedimientos y técnicas. Los protocolos LATIN y CME han logrado la capacitación personalizada de los operadores por parte de cardiólogos estadounidenses expertos en infarto agudo de miocardio - ST. El entrenamiento ha recalcado la importancia en la reperfusión rápida de la arteria comprometida con cortos tiempos de DTB. Ha fomentado técnicas estandarizadas, farmacología apropiada, manejo de trombos, estrategias de colocación de stents, uso de vasodilatadores intracoronarios, tratamiento del shock cardiogénico y el manejo de las demás lesiones coronarias no responsables del infarto agudo de miocardio. Se ha enfatizado en la deambulación, el egreso temprano del paciente y otras estrategias rentables.

8. LATIN ha contribuido a un cambio cultural y de actitud hacia el infarto agudo de miocardio con una deconstrucción sistemática y gradual de la práctica de "trombólisis durante la noche y $\mathrm{PCl}$ durante el día'.

9. Se han resaltado las fallas del sistema de salud, como el reembolso inadecuado y la falta de camas en las $\mathrm{UCI}$, y se ha comenzado una discusión nacional sobre las mismas.

\section{Conclusiones}

LATIN Colombia ha sido un programa transformador en el manejo del infarto agudo de miocardio en Colombia. Desde su origen, ha tratado cientos de pacientes mediante mecanismos de reperfusión basados en guías clínicas. El programa ha expandido colosalmente el acceso al tratamiento, con una desviación del manejo hacia el tratamiento urgente - $\mathrm{PCl}$ - considerado estándar para el infarto agudo de miocardio. El efecto de halo continúa mejorando el cuidado del infarto agudo de miocardio en toda la nación. Las mejoras en la tecnología de telemedicina son inminentes y éstas contribuirán a perfeccionar aun más los sistemas y las redes de Telemedicina. Todavía hay mucho trabajo por hacer para incrementar el número de pacientes tratados a partir de la cohorte de pacientes diagnosticados. Esta mejora requerirá un abordaje con enfoque múltiple, que involucre al personal medico, así como también a los servidores públicos y privados. Para el éxito de esta estrategia se requiere una política de salud pública incansable y el soporte inquebrantable de las sociedades de cardiología.

Finalmente, LATIN Colombia puede servir como un modelo ejemplar para mejorar el cuidado del infarto agudo de miocardio en regiones de África, Asia Sudoriental y Medio Oriente.

\section{Conflicto de intereses}

Ninguno.

\section{Financiación}

Ninguna.

\section{Bibliografía}

1. Levine GN, Bates ER, Blankenship JC, Bailey S, Bitti JA, Cercek B, et al. 2015 ACC/AHA/SCAl Focused Update on Primary Percutaneous Coronary Intervention for Patients With ST-Elevation Myocardial Infarction: An Update of the 2011 ACCF/AHA/SCAl Guideline for Percutaneous Coronary Intervention and the 2013 ACCF/AHA Guideline for the Management of ST-Elevation Myocardial Infarction. Circulation. 2016;133:1135-47.

2. Ibanez B, James S, Agewall S, Antunes MJ, Bucciarelli-Ducci C, Bueno $\mathrm{H}$, et al. ESC Guidelines for the management of acute myocardial infarction in patients presenting with ST-segment elevation: The Task Force for the management of acute myocardial infarction in patients presenting with ST-segment elevation of the European Society of Cardiology (ESC). Eur Heart J. 2018;39:117-9.

3. Oldroyd KG. Complete immediate revascularization of the patient with st-segment-elevation myocardial infarction is the new standard of care. Circulation. 2017;135:1571-3.

4. Acharya T, Salisbury AC, Spertus JA, Kennedy KF, Bhullar A, Kiran H, Reddy K, et al. In-Hospital Outcomes of Percutaneous Coronary Intervention in America's Safety Net. J Am Coll Cardiol: Cardiovascular Interventions. 2017;10:1475-85.

5. Jordan M, Caesar J. Improving door-to-needle times for patients presenting with ST-elevation myocardial infarction at a rural district general hospital. BMJ Qual Improv Rep. 2016;5, pii: u209049.w6736.

6. Reilly NM, Gunnarsson SI, Ciske B, Saari S, Leal M, Raval A, et al. In Hospital Triage of ST segment myocardial infarction patients results in favorable clinical outcomes and reduced ICU and Overall Hospital Length of Stay. Circulation. 2016;134:A16112. 
7. Kutcher MA. Door-to-balloon time as a process metric for treatment of st-segment elevation myocardial infarction. J Am Coll Cardiol: Cardiovascular Interventions. 2015;8:1975-7.

8. Patel N, Patel NJ, Thakkar B, Singh V, Arora S, Savani C, et al. Management strategies and outcomes of ST-segment elevation myocardial infarction patients transferred after receiving fibrinolytic therapy in the United States. Clin Cardiol. 2016;39:9-18.

9. Enezate TH, Omran J, Al-Dadah AS, Alpert M, Mahmud E, Patel $M$, et al. Comparison of outcomes of ST-elevation myocardial infarction treated by percutaneous coronary intervention during off-hours versus on-hours. J Am Coll Cardiol. 2017, pii:S0002-9149(17)31288-2.

10. Kodama N, Nakamura T, Yanishi K, Nakanishi N, Zen $\mathrm{K}$, Yamato $\mathrm{T}$, et al., Impact of Door-to-Balloon Time in Patients With ST-Elevation Myocardial Infarction Who Arrived by Self-Transport - Acute Myocardial InfarctionKyoto Multi-Center Risk Study Group. Circulation. 2017, http://dx.doi.org/10.1253/circj. CJ-17-0083.

11. Mentias A, Raza MQ, Barakat AF, Youseff D, Raymond R, Menon $\mathrm{V}$, et al. Effect of shorter door-to-balloon times over 20 years on outcomes of patients with anterior ST-elevation myocardial infarction undergoing primary percutaneous coronary intervention. J Am Coll Cardiol. 2017; S0002-9149, 31170-0.

12. Prasad A, Gersh BJ, Mehran R, Brodie BR, Brener SJ, Dizon $J M$, et al. Effect of ischemia duration and door-to-balloon time on myocardial perfusion in ST-segment elevation myocardial infarction. J Am Coll Cardiol: Cardiovascular Interventions. 2015;8:1966-74.

13. Regueiro A, Bosch J, Martín-Yuste V, Rosas A, Faixedas MT, Gomez JA, et al. Cost-effectiveness of a European ST-segment elevation myocardial infarction network: results from the Catalan Codi Infart network. BMJ Open. 2015;5:e009148.

14. Mayor S. Acute MI is initially misdiagnosed in nearly a third of patients, study finds. BMJ. 2016;354:i4713.

15. Schläpfer J, Wellens HJ. Computer-interpreted electrocardiograms. J Am Coll Cardiol. 2017;70:1183-92.

16. Steinhubl SR, Topol EJ. Moving from digitalization to digitization in cardiovascular care why is it important, and what could it mean for patients and providers? J Am Coll Cardiol. 2015;66(13):1489-96.

17. Madder R, Van Oosterhout S, Jacoby MJ, Stewart Collins AS, Borgman A, Mulder N, et al. Percutaneous coronary intervention using a combination of robotics and telecommunications by an operator in a separate physical location from the patient: an early exploration into the feasibility of teletesting (the REMOTEPCl study) Euro Intervention. 2017;12:1569-76.

18. Daniel H, Snyder Sulmasy L. Policy recommendations to guide the use of telemedicine in primary care settings: an american college of physicians position paper. Ann Intern Med. 2015;163:787-9.

19. Marcolino MS, Maia LM, Oliveira KA, Alkmim MB, Boersma E, Ribeiro Al, et al. Telemedicine application in the care of acute myocardial infarction patients: systematic review and metaanalysis. Circulation. 2016;134:A16282.

20. Jin Joo Park, Chang-Hwan Yoon, Jung-Won Suh, Young-Seok Cho, Tae-Jin Youn, In-Ho Chae, et al. Reduction of Ischemic Time for Transferred STEMI Patients Using a Smartphone Social Network System. J Am Coll Cardiol. 2016;68:1490-2.

21. Ho CK, Chen FC, Chen YL, Wang HT, Lee CH, Chung WJ, et al. Using a cloud computing system to reduce door-to-balloon time in acute ST-elevation myocardial infarction patients transferred for percutaneous coronary intervention. Biomed Res Int. 2017;2017:2963172.

22. Brunetti ND, De Gennaro L, Correale M, Santoro F, Caldarola P, Gaglione A, et al. Pre-hospital electrocardiogram triage with telemedicine near halves time to treatment in STEMI: A meta-analysis and meta-regression analysis of non-randomized studies. Int J Cardiol. 2017;232:5-11.

23. Tanguay A, Dallaire R, Hébert D, Bening F, Fleet R. Rural patient access to primary percutaneous coronary intervention centers is improved by a novel integrated telemedicine prehospital system. J Emerg Med. 2015;49:657-64.

24. Mehta S, Ferre A, Botelho R, Vega R, Bojanini R, Coral J, et al. Telemedicine increases access, accuracy \& cost-effectiveness of population based AMI care: results of the Latin America telemedicine infarct network (LATIN). Eur Heart J. 2017;38:41-3.

25. Mehta S, Ferre A, Botelho R, Vega R, Bojanini R, Coral J, et al. Telemedicine + STEMI systems of care: novel solution to massively expand access to AMI care in developing countries. Eur Heart J. 2017;38 suppl 1:3408.

26. Dallan L, Pazolini V, Matsuda C. Telemedicine as a landmark in the reduction of the door-to-balloon time in STEMls in distant areas in a developing country. J Am Coll Cardiol Intv. 2015;8 suppl 2:S15.

27. Macedo TA, de Barros E, Silva PG, Simões SA. Impact of Chest Pain Protocol with Access to Telemedicine on Implementation of Pharmacoinvasive Strategy in a Private Hospital Network. Telemed J E Health. 2016;22:549-52.

28. Matsuda C, Cade J, Janella B. Implementação do Sistema de Telemedicina no Atendimento Inicial dos Pacientes com Infarto Agudo do Miocárdio com Supradesnivelamento do Segmento ST na Zona Leste da Cidade de São Paulo. Paper presented at: 40th Congress of the Brazilian Society of Hemodynamics and Interventional Cardiology. Brazil: Brasilia; 2015.

29. Rekosz J, Kasznicka M, Kwiatkowska D, Mączyńska-Mazuruk R, Kotak K, Mierzejewska B, et al. Standard 12-lead electrocardiogram tele-transmission: Support in diagnosing cardiovascular diseases in operations undertaken by Warsaw-area basic medical rescue teams between 2009 and 2013. Cardiol J. 2015;22:675-82.

30. Yoculan A, Kim E, Eggington S, Au-Yeung A. Economic Value of STEMI program investment in Sao Paulo, Brazil. Value Health. 2015;18:A861.

31. Matsuda C, Cade J, Janella B. Implementação do Sistema de Telemedicina no Atendimento Inicial dos Pacientes com Infarto Agudo do Miocárdio com Supradesnivelamento do Segmento ST na Zona Leste da Cidade de São Paulo. Paper presented at: 40th Congress of the Brazilian Society of Hemodynamics and Interventional Cardiology;. Brazil: Brasilia; 2015.

32. Chan AW, Kornder J, Elliot H, Brown RI, Dorval JF, Charania J, et al. Improved survival associated with pre-hospital triage strategy in a large regional ST-segment elevation myocardial infarction program. JACC Cardiovasc Interv. 2012;5: 1239-46.

33. Brunetti ND, De gennaro L, Dellegrottaglie G, Di Giuseppe, Antonelli G, Di Biase M. All for one, one for all: Remote telemedicine hub pre-hospital triage for public Emergency Medical Service 1$1-8$ in a regional network for primary $\mathrm{PCl}$ in Apulia, Italy. Eur Res Telemed. 2014;3:9-15.

34. Rasmussen MB, Frost L, Stengaard C, Brohol- PetersenJU, Dot KK, Sondegaard HM, et al. Diagnostic performance and system delay using telemedicine for prehospital diagnosis in triaging and treatment of STEMI. Heart. 2014;100:711-5.

35. Sørensen JT, Terkelsen CJ, Nørgaard BL, Trautner S, Hansen TM, Botker HE, et al. Urban and rural implementation of prehospital diagnosis and direct referral for primary percutaneous coronary intervention in patients with acute ST-elevation myocardial infarction. Eur Heart J. 2011;32:430-6.

36. Sanchez-Ross M, Oghlakian G, Maher J, Patel B, Mazza V, Hom D, et al. The STAT-MI (ST-Segment Analysis Using Wireless Technology in Acute Myocardial Infarction) trial improves outcomes. JACC CardiovascInterv. 2011;4:222-7. 